\title{
Construção de Gráficos de Barras no Ensino Fundamental da Educação de Jovens e Adultos
}

\author{
Construction of bar graphs in Elementary Education of Youth and Adult Education
}

\author{
Izauriana Borges Lima; ; Ana Côelho Vieira Selva*a
}

aUniversidade Federal de Pernambuco, Programa de Pós-Graduação em Educação Matemática e Tecnológica. PE, Brasil.

*E-mail: anacvselva@gmail.com

\begin{abstract}
Resumo
A importância do ensino da construção de gráficos encontra justificativa na necessidade de desenvolver habilidades específicas ligadas tanto aos seus elementos estruturantes, como a adequação dos dados ao tipo de gráfico e a definição da escala a ser utilizada, quanto à valorização dessa representação como meio de transmitir uma informação. Sendo assim, o objetivo deste estudo foi analisar o desempenho de estudantes do Ensino Fundamental da EJA do município de Jaboatão dos Guararapes-PE em atividades de construção de gráficos de barras a partir de uma tabela. Participaram do estudo 88 estudantes do Ensino Fundamental, sendo 44 do Módulo III e 44 do Módulo V. Os resultados evidenciaram a ausência de elementos estruturantes nos gráficos de barras construídos, como o título, entretanto, a maioria apresentou a descrição das categorias e frequências e o uso da linha de base. A construção da escala foi a dificuldade mais evidente. Apesar das dificuldades identificadas verificamos efeito positivo da escolaridade tendo os estudantes do Módulo V construído mais gráficos quando solicitados do que o grupo de estudantes com menor escolaridade. A escolaridade também favoreceu o aprimoramento dos gráficos de barras construídos. Os resultados parecem indicar a necessidade um trabalho mais sistematizado em sala de aula voltado para questões fundamentais da construção de gráficos refletindo-se, sobretudo, a inclusão de elementos básicos necessários para a transmissão da informação e a adequação da escala, pois o desenvolvimento da habilidade de construir uma representação gráfica é parte do componente cognitivo na perspectiva do letramento estatístico do adulto.
\end{abstract}

Palavras-chave: Letramento. Educação de Jovens e Adultos. Construção. Gráficos de Barras.

\begin{abstract}
The importance of teaching the construction of graphs is justified by the need to develop specific skills linked to both its structuring elements, such as the adequacy of the data to the type of graph and the definition of the scale to be used, as to the valorization of this representation as a means of transmitting a information. Therefore, the objective of this study was to analyze the performance of students of the EJA Elementary School in the city of Jaboatão dos Guararapes-PE in activities of building bar graphs from a table. 88 elementary school students participated in the study, 44 from Module III and 44 from Module V. The results showed the absence of structuring elements in the constructed bar graphs, such as the title, however, most presented the description of the categories and frequencies and the use of the baseline. The construction of the scale was the most evident difficulty. Despite the difficulties identified, we verified a positive effect of schooling, with Module V students building more graphics when requested than the group of students with less education. Schooling also favored the improvement of the built bar graphs. The results seem to indicate the need for more systematic work in the classroom focused on fundamental issues of graph construction, reflecting, above all, the inclusion of basic elements necessary for the transmission of information and the adequacy of the scale, as the development of the skill building a graphic representation is part of the cognitive component from the perspective of adult statistical literacy.
\end{abstract}

Keywords: Literacy. Youth and Adult Education (EJA). Construction. Bar Graphs.

\section{Introdução}

Esta pesquisa, desenvolvida a partir de um estudo de Doutorado, teve o objetivo de analisar o desempenho de 88 estudantes dos anos iniciais e anos finais do Ensino Fundamental da modalidade de Educação de Jovens e Adultos (EJA), matriculados na rede pública do município de Jaboatão dos Guararapes - Pernambuco, em atividades de construção de gráficos de barras a partir de dados contidos em tabelas.

Os estudantes da Educação de Jovens e Adultos (EJA) caracterizam-se por decorrentes processos históricos, políticos, educacionais e sociais, como a exclusão do sistema regular de ensino, a constituição de singularidades socioculturais e a superação de dificuldades pessoais num contexto de mundo mediado pela linguagem escrita, que trazem repercussões para o processo de ensino e de aprendizagem e que devem ser consideradas na educação formal. Alia-se a isto, a escassez de pesquisas na EJA, em especial na área de Matemática, no cenário nacional e internacional, bem como os altos índices de abandono e baixos índices de aprendizagem documentados por órgãos de educação municipal, estadual e nacional.

Segundo estudo publicado pelas equipes de profissionais do Instituto Paulo Montenegro e Ação Educativa (2016), em que foram entrevistadas 2.002 pessoas entre 15 e 64 anos de idade residentes em zonas urbanas e rurais de todas as regiões do país, utilizando-se articuladamente testes cognitivos e questionários de contextualização sócio demográfica, econômica, cultural e educacional, não houve avanços no letramento e numeramento da população adulta brasileira nos últimos anos. Os resultados indicaram que $27 \%$ das pessoas 
foram classificadas como analfabetas funcionais, mantendose "estável na comparação com os resultados obtidos em 2011 na última edição do Inaf Brasil” (Instituto Paulo Montenegro e Ação Educativa, 2016), representando uma imobilidade severa na melhoria das habilidades dos adultos mesmo quando escolarizados.

Durante muito tempo prevaleceu a concepção de que os jovens e adultos analfabetos ou pouco escolarizados não possuíam conhecimentos sistematizados da Matemática e que, portanto, caberia à escola a missão de garantir os conteúdos necessários para que pudessem se apropriar dos conhecimentos desta área. Entretanto, jovens e adultos analfabetos resolvem cotidianamente situações simples nas quais os conhecimentos matemáticos são acionados, tanto nas suas atividades pessoais quanto nas atividades profissionais. Apesar da natureza prática da mobilização de conhecimentos matemáticos na resolução de problemas reais, tais conhecimentos, geralmente, não são generalizados para situações mais complexas.

A necessidade de mobilizar o conhecimento já elaborado para situações ainda não vivenciadas na prática é extremamente importante para auxiliar jovens e adultos a enfrentarem desafios cada vez mais amplos. Assim sendo, a escola se caracteriza como local indispensável para auxiliar os estudantes a aprenderem a utilizar procedimentos mais econômicos, mais rápidos e precisos, adequados a uma variedade de situações, bem como dar as condições de aquisição de novos conhecimentos matemáticos.

Segundo Soek, Haracemiv \& Stoltz (2009) quando jovens e adultos passam a ter acesso a conceitos científicos trabalhados no universo escolar, a partir da interação social, desenvolvem funções psicológicas superiores como "atenção voluntária, memória voluntária, linguagem, consciência, comportamento intencional, raciocínio abstrato, pensamento generalizante, afetividade propriamente humana e outras".

Assim sendo, devemos reconhecer que a escola tem muito a contribuir com os processos de aprendizagem e ensino dos jovens e adultos pelo papel que cumpre na transmissão e consolidação de valores e conhecimentos socialmente reconhecidos, constituindo-se local propício à valorização do conhecimento prévio que estes detêm e elevá-lo a um nível científico capaz de lhes dar explicações mais avançadas acerca da realidade vivida e ampliar as possibilidades de atuação na mesma.

Justificamos a investigação na área da Matemática, especificamente no conteúdo das representações dos dados estatísticos contido na unidade temática Probabilidade $e$ estatística (BNCC, 2017) ${ }^{1}$, pelo importante papel social que a apropriação dos conhecimentos subjacentes à essas representações assumem nas práticas de leitura e transmissão de informações através de gráficos apresentados em mídias digitais e impressas e pela necessidade de desenvolver as habilidades inerentes às condições de construir uma representação gráfica. Neste sentido, esta unidade temática ganha bastante relevância na atualidade na medida em que a acessibilidade a dados de natureza quantitativa exige tanto a compreensão de elementos próprios da Estatística, quanto a reflexão das ideias contidas nestas informações, sendo esses conhecimentos fundamentais para o letramento estatístico e para a construção de uma cidadania ativa.

\section{Letramento Estatístico}

De acordo com Gal (2002, p.2-3), o letramento estatístico refere-se amplamente a dois componentes inter-relacionados:

(a) Habilidades para interpretar e avaliar criticamente informações estatísticas, relacionadas a dados, argumentos ou fenômenos estocásticos, que podem ser encontrados em diversos contextos e,

(b) Habilidades de discutir ou comunicar suas reações a tais informações estatísticas, como a compreensão do significado da informação, suas opiniões sobre as implicações desta informação, ou suas preocupações quanto à aceitabilidade de determinadas conclusões.

Gal (2002) propôs ainda um modelo de letramento estatístico do adulto que envolve dois componentes interrelacionados: o cognitivo e o disposicional. O componente cognitivo é composto por cinco elementos: a alfabetização, o conhecimento estatístico, o conhecimento matemático, o conhecimento do contexto e a formulação de questões críticas que possam ser feitas. Já o componente disposicional é composto por dois elementos: as crenças e atitudes e o posicionamento crítico.

$\mathrm{O}$ primeiro diz respeito às atitudes e às crenças das pessoas, que moldam suas visões de mundo; e o segundo diz respeito à postura crítica, que nada mais é do que a propensão para um comportamento questionador diante de informações estatísticas. (Cazorla \& Santana, 2010, p.12).

Gal (2002) ressalta que os componentes do modelo proposto não devem ser vistos como elementos fixos e separados, mas como um contexto dependente da dinâmica do conhecimento e das disposições, que juntos ativam o comportamento favorável ao letramento estatístico. A compreensão e a interpretação da informação estatística requerem conhecimentos não só estatísticos, mas também de outras naturezas como o conhecimento matemático e contextual. Depois de entendida a informação estatística, a capacidade para pensar criticamente frente às informações depende de elementos adicionais como a habilidade de avaliar questões e tomar uma postura crítica que por sua vez é apoiado por certas crenças e atitudes.

Desta forma, podemos considerar o letramento estatístico situado como competência central que envolve várias habilidades necessárias para que os sujeitos possam refletir em torno das informações estatísticas e atuar ativamente na

1 A Base Nacional Comum Curricular (BNCC) é um documento de caráter normativo que define o conjunto orgânico e progressivo de aprendizagens essenciais que todos os alunos devem desenvolver ao longo das etapas e modalidades da Educação Básica. (Brasil, 2017). 
sociedade a partir destas informações. Nesta perspectiva, ser estatisticamente letrado ou exercer cidadania estatística é condição sumariamente importante para a formação do estudante jovem e adulto, na medida em que situações de exclusão ou de precariedade de acesso aos bens produzidos cientificamente podem ser superadas a partir do domínio do conhecimento estatístico.

Enfatizamos ainda a habilidade de construir uma representação como parte do componente cognitivo na perspectiva do letramento estatístico, ou seja, estar estatisticamente letrado significa também ser capaz de comunicar dados ou resumos estatísticos por meio de representações gráficas. (Gal, 2002). Assim sendo, contribuem para a efetividade do exercício da cidadania, tanto o desenvolvimento das habilidades de leitura e interpretação de gráficos quanto o desenvolvimento das habilidades para construir estas representações.

\section{Construção de Gráficos}

Construir gráficos constitui um dos objetivos de aprendizagem presente em todos os documentos nacionais do currículo para a Educação de Jovens e Adultos. (Brasil, 2001, 2002). A importância do ensino da construção de gráficos encontra justificativa na necessidade de desenvolver habilidades específicas ligadas tanto aos seus elementos estruturantes (como a adequação dos dados ao tipo de gráfico e a definição da escala a ser utilizada, por exemplo), quanto à valorização dessa representação como meio de transmitir uma informação.

Além dos aspectos da regularidade envolvidos na construção dos gráficos de barras, que devem ser considerados, ressaltamos atenção especial aos elementos constitutivos que dão sentido à representação, tais como o tema abordado, seus elementos estruturais, a inclusão de informações adicionais e a proporção da escala, pois esses elementos podem ter influências diferentes para os estudantes e ao mesmo tempo, impactam na compreensão dos dados representados. Nesse sentido, torna-se pertinente ainda buscar compreender estes elementos a partir de estudos sobre as atividades de leitura e interpretação de gráficos buscando as possíveis articulações com a tarefa de produzir um novo gráfico.

Nas tarefas de construção de gráficos vários aspectos devem ser considerados, tanto os relativos às aprendizagens decorrentes das situações de ensino promovidas pela escola como os elementos inerentes à estruturação e aos objetivos da representação gráfica, transmitir ou comunicar uma informação; bem como as experiências extraescolares que por alguma razão possam interferir na representação realizada.

O desenvolvimento de competências necessárias para que se possa construir gráficos está relacionado ao raciocínio estatístico, que segundo Garfield (2002), pode ser definido como a maneira como as pessoas lidam com ideias estatísticas e como dão sentido à informação estatística (informações baseadas em conjuntos de dados, representações gráficas e resumos estatísticos). Mais especificamente, o raciocínio sobre os dados e o raciocínio sobre representação dos dados, nos permite focar nas representações dos dados estatísticos e consequentemente analisar as questões relativas à construção de gráficos e a compreender as relações que estão em jogo quando um sujeito se depara com a necessidade de criar gráficos. Ou seja, reconhecer ou categorizar dados como quantitativo ou qualitativo, discreto ou contínuo; e saber por que o tipo de dado leva a um determinado tipo de tabela, gráfico ou medida estatística (raciocínio sobre os dados) e reconhecer as suas características gerais e compreender como os gráficos podem ser apropriados para melhor representar um determinado conjunto de dados (raciocínio sobre representações de dados).

Segundo Friel, Curcio \& Bright (2001) as orientações didáticas sobre como produzir diferentes tipos de gráficos são oferecidas com pouca atenção nas escolas, sendo essa uma das razões para as dificuldades no desenvolvimento da habilidade de construir gráficos. Entretanto, enfatizamos que tanto a variabilidade de tipos de gráficos, nas quais as atividades podem ser focadas na análise exploratória dos dados (raciocínio sobre os dados); quanto o aprofundamento das características estruturantes de cada tipo de gráfico, por meio de atividades que envolvam a sua construção (raciocínio sobre representações de dados), podem cooperar com o desenvolvimento do raciocínio estatístico possibilitando consequentemente maiores chances de êxito em tarefas desse tipo.

Considerando o reconhecimento das características relativas ao raciocínio sobre representações de dados estudos têm mostrado muitas dificuldades em relação aos elementos considerados essenciais na construção de gráficos. (Morais, 2010; Lima, 2010; Alcântara, 2012; Santos, 2014). Tais dificuldades podem estar associadas às dificuldades dos professores em desenvolverem práticas de construção de gráficos em contextos mais significativos ou à prioridade em se trabalhar a partir de gráficos já construídos. (Friel, Curcio \& Bright, 2001; Martins \& Carvalho, 2018).

Alguns destes estudos têm destacado dificuldades por parte dos estudantes da EJA ao serem solicitados a construírem gráficos (Lima, 2010; Alcântara, 2012; Santos, 2014). Dificuldades com a adequação da escala na construção de gráficos foram observadas em Lima (2010), em que apenas $12,1 \%$ dos gráficos construídos apresentavam a escala proporcional, mesmo tendo sido solicitada a construção em malha milimetrada. Também foram observadas dificuldades em incluir determinados elementos estruturantes do gráfico, como o título, a nomeação dos eixos e a representação do zero na escala. As análises dos resultados apontaram para a necessidade de refletir as práticas adotadas para o ensino da Estatística, especificamente quanto aos gráficos, pois os baixos desempenhos apresentados pelos estudantes revelaram as fragilidades em atender os critérios necessários à representação adequada das informações. 
Alcântara (2012) ao analisar os relatos de experiência de professores do ProJovem Campo indicou que atividades com construção de gráficos e tabelas foram trabalhados no Programa, mas o trabalho com conceitos importantes como escala e outros aspectos necessários para a compreensão de gráficos e tabelas, como o título, não foram muito abordados nesses relatos de experiência. Nos extratos apresentados e, posteriormente, analisados pela pesquisadora, observaramse erros na construção da escala, falta dos descritores e do ponto zero, por exemplo. Entretanto, chamamos atenção para a ausência de informação sobre como foram conduzidas as tarefas de construção de gráficos, a falta de material adequado para que os estudantes confeccionassem os gráficos e a ausência de correções feitas pelos educadores nas tarefas feitas por seus alunos.

Santos (2014) analisou experiências de atividades para o ensino de construção de gráficos sem e com o uso do computador nas aulas de Matemática em escolas núcleos do ProJovem Urbano ${ }^{2}$ em Recife e concluiu que as dificuldades dos estudantes em construírem gráficos com lápis e papel tinham relação com aspectos conceituais que poderiam ter sido mais evidenciados pelas docentes, caso tivessem focado em discutir sobre as produções dos alunos e se tivessem estimulado os alunos a interpretarem os gráficos construídos, evidenciando assim tanto a necessidade de refletir os aspectos específicos da tarefa de construir gráficos, quanto a articulação entre as tarefas de interpretar e construir.

Assim sendo, podemos considerar algumas implicações para os processos de ensino quanto à construção de gráficos como a análise da natureza dos dados que se deseja representar e a maneira adequada de representá-los; e a ênfase quanto ao reconhecimento das características gerais da representação e quanto à forma e informações a serem destacadas. Neste sentido, nosso estudo se debruçou sobre as atividades de construção de gráficos de barras a partir de dados apresentados em tabelas, nas quais foram analisados os estruturantes incluídos na construção e as dificuldades mais evidenciadas.

\section{Material e Métodos}

O objetivo geral deste estudo foi analisar o desempenho de estudantes ao longo do Ensino Fundamental da modalidade de EJA em atividades de construção de gráficos de barras. Especificamente, comparamos o desempenho dos estudantes em diferentes fases de escolarização (anos iniciais e anos finais do Ensino Fundamental) na construção de gráficos de barras; analisamos os avanços e as dificuldades dos estudantes na construção de gráficos de barras; e verificamos os gráficos elaborados pelos estudantes considerando seus elementos constituintes (título, descrição e nomeação de categorias e frequências, linha de base e escala).

Participaram deste estudo 88 estudantes da EJA matriculados nos módulos III e V, correspondente ao último ano dos anos iniciais e anos finais do Ensino Fundamental respectivamente, sendo 44 estudantes do Módulo III e 44 estudantes do Módulo V. Os participantes foram solicitados a resolver duas atividades de construção de um gráfico de barras a partir de uma tabela.

As coletas foram realizadas em cinco escolas e contou com a participação de sete turmas, sendo quatro turmas do Módulo III e três turmas do Módulo V. Os participantes dos anos iniciais pertenciam a quatro escolas, enquanto que os do Módulo $\mathrm{V}$ eram todos da mesma escola. Essa diferença na quantidade de escolas reflete a realidade da organização da EJA no município, que tem turmas pequenas dos anos iniciais e distribuídas em diferentes escolas e nos anos finais apresenta escolas polo, com maior número de turmas de anos finais. Veja no Quadro 1 a distribuição das turmas por escola e a quantidade de participantes em cada turma.

Figura 1 - Distribuição dos participantes por escola e módulo

\begin{tabular}{|c|c|c|c|}
\hline Escola & Módulo & Turma & $\mathrm{N}^{\circ}$ de Alunos \\
\hline Escola A & M III & Única & 7 \\
\hline Escola B & M III & Única & 11 \\
\hline Escola C & M III & Única & 11 \\
\hline Escola D & M III & Única & 15 \\
\hline \multirow{3}{*}{ Escola E } & \multirow{3}{*}{ M V } & 1 & 16 \\
\hline & & 2 & 12 \\
\hline & & 3 & 16 \\
\hline
\end{tabular}

\section{Fonte: Os autores.}

A aplicação do teste foi realizada em um encontro por turma, com duração média de 2 horas.

\subsection{Atividades de construção de gráfico de barras a partir de uma tabela}

A seguir serão apresentadas as atividades de construção de um gráfico de barras a partir de uma tabela elaboradas para este estudo. Destacamos que os dados contidos nas tabelas têm valores percentuais referentes a uma continuidade cuja soma total é de $100 \%$ e as categorias são nominais. Também consideramos a necessidade de abordar temas reais do cotidiano que pudessem despertar o interesse ou a curiosidade dos participantes, deste modo, os dados foram extraídos de informações recuperáveis em pesquisa de ampla divulgação pela internet cujas fontes fossem confiáveis.

\section{Atividade C-1}

A atividade C-1, ilustrada na Figura 2, indica a distribuição

2 O Programa Nacional de Inclusão de Jovens - Projovem Urbano é um programa educacional destinado a jovens residentes em áreas urbanas que, por diversos motivos, foram excluídos da escolarização, com o objetivo de reintegrá-los ao processo educacional, elevar sua escolaridade e promover sua formação cidadã e qualificação profissional, por meio de curso com duração de dezoito meses. O Projovem Urbano destina-se a jovens de 18 a 29 anos que saibam ler e escrever, mas não tenham concluído o Ensino Fundamental. Podem aderir ao Projovem Urbano as secretarias de educação dos estados e do Distrito Federal e prefeituras dos municípios com população igual ou superior a cem mil habitantes (informações disponíveis no site do fnde.gov.br). 
percentual do uso de água no Brasil segundo levantamento da Agência Nacional de Águas (ANA) em 2012. Os dados estão representados por uma tabela simples e o estudante é solicitado a construir um gráfico de barras a partir dos dados apresentados na tabela.

\section{Atividade}

Observe a tabela abaixo referente à distribuição do uso da água no Brasil de acordo com o levantamento da Agência Nacional de Águas (ANA) em 2012:

Figura 1- Atividade C-1 - Construção de gráfico de barras sobre a distribuição do uso da água no Brasil de acordo com o levantamento da Agência Nacional de Águas (ANA) em 2012. Tabela: Distribuição do uso da água no Brasil

\begin{tabular}{c|c}
\hline Setores & Porcentagem \\
\hline Agricultura (irrigação) & $72 \%$ \\
\hline Pecuária & $11 \%$ \\
\hline Consumo urbano & $9 \%$ \\
\hline Indústria & $7 \%$ \\
\hline Consumo rural & $1 \%$ \\
\hline
\end{tabular}

Fonte: ANA, 2012.

a) Construa um gráfico de barras abaixo a partir dos dados apresentados na tabela:

\section{Atividade C-2}

A atividade C-2, ilustrada na Figura 2, indica os valores percentuais da mortalidade por tipo de acidentes entre a população brasileira de 0 a 14 anos de idade divulgados pelo Ministério da Saúde em 2012. Os dados estão representados por uma tabela simples e o estudante é solicitado a construir um gráfico de barras a partir dos dados apresentados na tabela.

\section{Atividade}

Observe na tabela a seguir os dados relacionados a mortalidade por acidentes entre 0 e 14 anos de idade segundo dados divulgados pelo Ministério da Saúde em 2012.

Figura 2 - Atividade C-2 - Construção de gráfico de barras sobre a mortalidade por acidentes entre 0 e 14 anos de idade segundo dados divulgados pelo Ministério da Saúde em 2012.

Tabela: Mortalidade por acidentes 0-14a - 2012

\begin{tabular}{c|c}
\hline Acidentes & Porcentagem \\
\hline Trânsito & $40 \%$ \\
\hline Afogamento & $25 \%$ \\
\hline Sufocação & $16 \%$ \\
\hline Queimaduras & $6 \%$ \\
\hline Queda & $5 \%$ \\
\hline Outros & $8 \%$ \\
\hline
\end{tabular}

Fonte: Ministério da Saúde. Datasus (2012).

a) Construa um gráfico de barras abaixo a partir dos dados apresentados na tabela:

\section{Resultados e Discussão}

Analisamos o desempenho apresentado pelos participantes considerando o módulo de escolaridade em que estavam matriculados, ou seja, comparamos o desempenho dos estudantes do Módulo III e do Módulo V nas atividades de construção de gráficos. Consideramos para efeito do cálculo do percentual de acerto nas atividades de construção o quantitativo total de gráficos construídos. Destacamos que consideramos que houve a construção de um gráfico quando, no mínimo, havia o desenho das barras nos eixos, respeitando ou não a linha de base, com suas respectivas frequências, ainda que sem proporcionalidade entre elas.

O percentual geral de acerto nas atividades de construção por grupo de escolaridade foi de $60 \%$ para o Módulo III e $86 \%$ para o Módulo V. Para saber se essas diferenças foram significativas, foi realizada uma análise de variância (ANOVA) do programa Statistical Packedge for the Social Sciences (SPSS), na qual verificamos que os estudantes do Módulo V apresentaram desempenho significativamente melhor do que os estudantes do Módulo III nas atividades de construir (F, gl1=7,974; $\mathrm{p}<0,006$ ).

Também analisamos o percentual de gráficos construídos em cada tarefa proposta (C-1 e C-2), considerando as especificidades de cada uma delas, por grupo de escolaridade. Assim sendo, comparamos as diferenças por atividade de construção solicitada considerando a temática abordada, uma tratava sobre distribuição do uso da água (C-1) e a outra sobre a mortalidade por acidentes (C-2). O percentual de estudantes, por módulo, que realizaram a atividade de construção em cada situação proposta (C-1 e C-2) pode ser visto no Quadro 2.

Quadro 2 - Percentual de gráficos construídos por atividade e por módulo

\begin{tabular}{|c|c|c|}
\hline \multirow{2}{*}{ Módulos } & \multicolumn{2}{|c|}{ Atividades } \\
\cline { 2 - 3 } & C-1 Água & C-2 Mortalidade \\
\hline M III & $54 \%$ & $66 \%$ \\
\hline M V & $84 \%$ & $89 \%$ \\
\hline Total & $69 \%$ & $77 \%$ \\
\hline
\end{tabular}

Considerando o total de gráficos construídos por tarefa (C-1 e C-2), os estudantes, em geral, construíram mais gráficos na proposição dos dados relacionados à mortalidade por acidentes entre 0 e 14 anos de idade (C-2) do que referente à distribuição do uso da água no Brasil de acordo com o levantamento da Agência Nacional de Águas (ANA) em 2012 (C-1). Esse mesmo comportamento foi observado considerando os módulos, sendo a diferença maior quando se observa o Módulo III. Ou seja, foi mais difícil para os estudantes do Módulo III construírem gráficos de barras na situação C-1 comparada à situação $\mathrm{C}-2$, do que para os estudantes do Módulo V.

Outra análise realizada foi verificar os percentuais de estudantes que construíram ao menos um gráfico, uma vez que foram solicitados a construírem dois. A partir dos resultados obtidos em relação aos grupos de estudantes que construíram gráficos (pelo menos um dos dois gráficos propostos), observamos que $68 \%$ dos alunos dos anos iniciais do Ensino Fundamental construíram ao menos um gráfico e $89 \%$ dos alunos dos anos finais do Ensino Fundamental construíram ao menos um gráfico. Sendo assim, ratificamos a escolarização como um aspecto positivo nas atividades de construção de 
gráficos entre os segmentos de ensino da EJA. Entretanto, é importante chamar atenção para o fato de que os gráficos construídos apresentavam muitas inadequações e a ausência de elementos estruturantes importantes (como a nomeação de eixos e o título).

Os elementos incluídos nos gráficos construídos pelos estudantes foram a descrição das frequências, a descrição das categorias, o uso da linha de base, a organização entre a altura das barras (relação de diferenciação entre a altura das barras considerando os valores das suas frequências como tentativa de representar a proporcionalidade da escala e organização da disposição das barras apenas por ordenação) e a proporcionalidade da escala. Na tabela a seguir podem ser visualizados os elementos incluídos nos gráficos de barras construídos pelos estudantes dos módulos III e V em ambas as atividades de construção (C-1 e C-2).

Quadro 3 - Percentual de elementos incluídos nos gráficos construídos por módulo e atividade

\begin{tabular}{|c|c|c|c|c|c|c|c|}
\hline \multirow{2}{*}{ Elementos } & \multicolumn{2}{|c|}{ M III } & \multicolumn{2}{|c|}{ M V } & \multicolumn{2}{|c|}{ Total } & \\
\cline { 2 - 8 } & $\mathbf{C 1}$ & $\mathbf{C 2}$ & $\mathbf{C 1}$ & $\mathbf{C 2}$ & $\mathbf{C 1}$ & $\mathbf{C 2}$ & Total \\
\hline $\begin{array}{c}\text { Descrição das } \\
\text { frequências }\end{array}$ & 100 & 100 & 100 & 100 & 100 & 100 & 100 \\
\hline $\begin{array}{c}\text { Descrição das } \\
\text { categorias }\end{array}$ & 75 & 59 & 68 & 67 & 70 & 63 & 67 \\
\hline Linha de base & 71 & 72 & 81 & 85 & 77 & 79 & 78 \\
\hline $\begin{array}{c}\text { Ordenação das } \\
\text { barras }\end{array}$ & 42 & 55 & 54 & 69 & 49 & 63 & 57 \\
\hline $\begin{array}{c}\text { Tentativa de } \\
\text { representar a } \\
\text { proporcionalidade }\end{array}$ & 21 & 7 & 24 & 13 & 23 & 10 & 16 \\
\hline $\begin{array}{c}\text { Proporcionalidade } \\
\text { da escala }\end{array}$ & 4 & - & - & - & 2 & - & 1 \\
\hline
\end{tabular}

De modo geral, nenhum dos gráficos construídos recebeu o título ou recebeu a nomeação dos eixos, dois elementos estruturantes importantes à compreensão de um gráfico e que teriam pertinência de uso no caso das tarefas propostas. Todos os gráficos construídos apresentaram a descrição das frequências, pois o critério mínimo para ter sido considerado como gráfico era ter o desenho das barras nos eixos com as respectivas frequências, como já dito. A maior parte dos gráficos construídos apresentou a descrição das categorias nominais, $67 \%$, o uso da linha de base para a construção das barras, $78 \%$, e ordenaram as barras por seriação decrescente, $57 \%$.

A construção da escala considerando a proporcionalidade entre as frequências foi a maior dificuldade observada, confirmando estudos da área. (Albuquerque, 2010, Lima, 2010, Santos, 2014). Apenas um estudante dos anos iniciais utilizou a régua para estabelecer intervalo proporcional no eixo das frequências conseguindo construir corretamente a escala na atividade de C-1 (Água), entretanto, como já mencionado, tentativas de diferenciar a altura das barras foram observadas.
Analisamos as estratégias que os estudantes utilizaram para delimitar o tamanho das barras e observamos que 16\% consideraram a natureza proporcional entre as frequências, ou seja, embora os estudantes não conseguissem adequar corretamente a escala, tentaram representar a natureza proporcional, mesmo que de maneira grosseira, a altura das barras comparando os valores das frequências entre si e $57 \%$ apenas ordenaram as barras por seriação decrescente seguindo a tabela da tarefa.

A partir dos gráficos construídos observamos que a inclusão da descrição das categorias e o uso da linha de base estavam presentes, independente do gráfico a ser construído. Entretanto, verificamos diferenças nos percentuais em torno da tentativa de diferenciar a altura das barras entre as tarefas de construção C-1 e C-2, sendo mais fácil representar a natureza proporcional da escala na atividade de construção de gráfico de barras C-1 do que C-2.

Descrição das frequências e categorias e uso da linha de base

Apesar de todos os gráficos de barras construídos apresentarem a descrição das frequências, esta descrição por si só não garantia a compreensão do assunto tratado, tornando-se necessária a inclusão de outros elementos como a nomeação dos eixos, a descrição das categorias e a produção de um título, por exemplo. Veja o extrato a seguir, Figura 3, no qual as frequências são registradas, mas é impossível fora do contexto da tarefa solicitada saber à que se referem os números apresentados.

Figura 3 - Gráfico de barras construído por um estudante do Módulo V. Sujeito 8 - Turma B - M V. Atividade C-1 acima

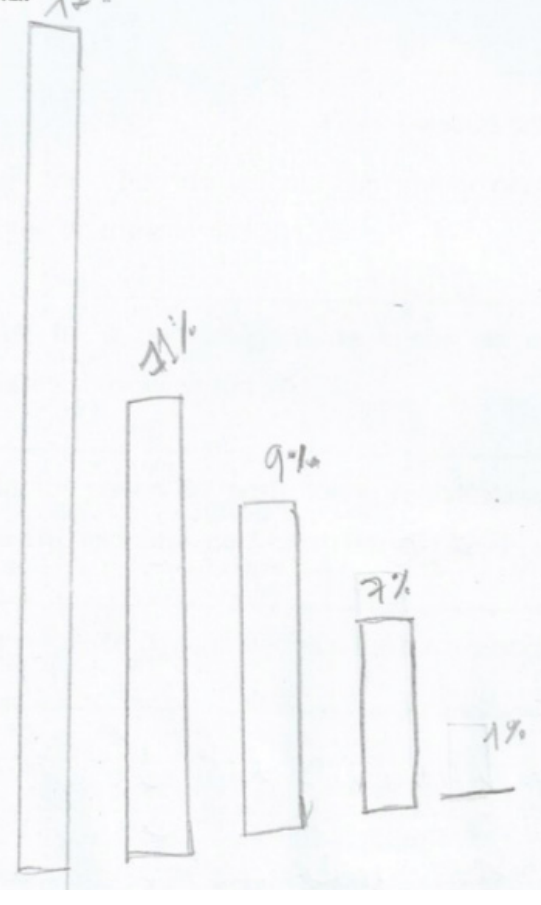

2 As colunas não somam $100 \%$, pois os elementos incluídos são categorias mutuamente exclusivas, ou seja, ocorreram simultaneamente na medida em que um mesmo sujeito poderia usar mais de um deles no mesmo gráfico construído. 
Além do registro das frequências também estavam presentes, na maior parte dos gráficos de barras construídos, a descrição das categorias (67\%), o que significa que a maioria dos gráficos contemplavam dois estruturantes simultaneamente (frequências e categorias). Entretanto, não podemos deixar de considerar que eles já estavam dispostos na tabela, o que facilitava a conversão para uma nova representação. Também é importante destacar que embora as categorias tenham sido registradas a inclusão de outros elementos continuava sendo necessária ao gráfico para auxiliar a sua leitura. A Figura 4 ilustra um gráfico construído em que há a descrição das categorias nominais.

Figura 4 - Gráfico de barras construído por uma estudante do Módulo V. Sujeito 7 - Turma C - M V. Atividade C-1

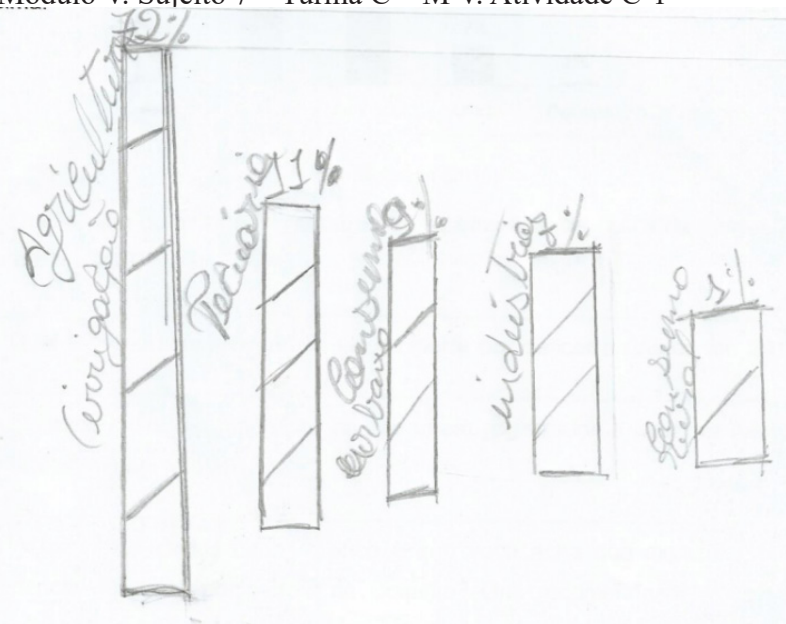

Observe que a presença de outros estruturantes como a nomeação dos eixos ou a produção de um título auxiliariam a compreender as variáveis qualitativas, pois só é possível saber que se referem aos setores que usam a água distribuída para consumo no Brasil no contexto da tarefa realizada.

$\mathrm{Na}$ mesma produção, observe ainda que a estudante não usa uma linha de base para estabelecer o ponto inicial da escala. Mesmo tendo sido observado que a maior parte dos gráficos construídos teve a linha de base estabelecida, $78 \%$, não podemos deixar de mencionar a inviabilidade da construção adequada da escala sem que todas as frequências se iniciem na mesma base.

Neste sentido, enfatizamos o papel da escola no desenvolvimento das tarefas de construção de gráficos, pois é esperado que os estudantes da EJA até o final do Ensino Fundamental apresentem os dados de modo adequado e reconheçam os elementos de um gráfico conseguindo produzilo de maneira correta. (Pernambuco, 2012). É importante que compreendam que o gráfico deve ser lido por si próprio, pois se caracteriza como suporte representacional rápido e sucinto na divulgação de ideias e informações, portanto, ao produzir um, é importante que sejam capazes de avaliar sua clareza levando em consideração a sua estrutura e finalidade.

Como já mencionamos anteriormente verificamos apenas um gráfico construído com a escala calibrada através do uso da régua, material disponibilizado para que os estudantes utilizassem durante a aplicação dos testes deste estudo. Verificamos que um estudante do Módulo III correlacionou a décima parte de cada frequência para poder adequar a escala em centímetros e milímetros (unidade de medida e subunidade de medida da régua) como mostra a Figura 5.

Figura 5 - Gráfico de barras construído por um estudante do Módulo III. Sujeito 5 - Turma A - M III. Atividade C-1

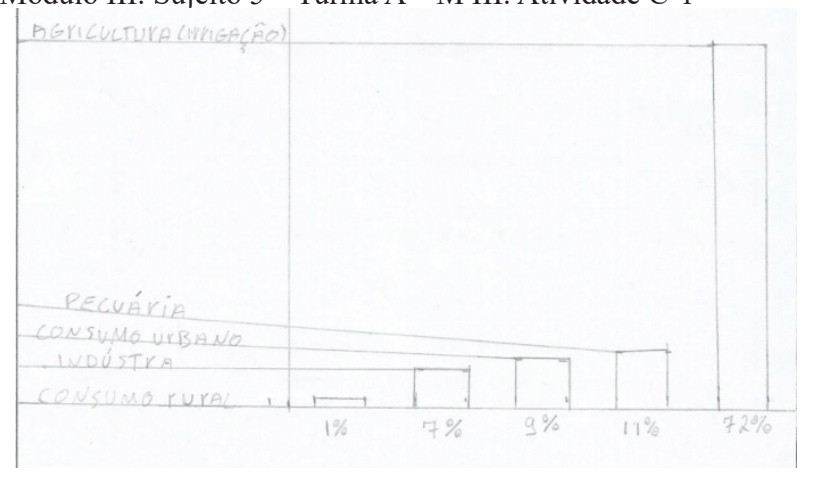

Percebemos que a escala utilizada por ele obedeceu ao mesmo critério para todas as frequências: dividir por 10 o valor da frequência e correlacionar o resultado da divisão com a medida em centímetros e milímetros na régua. Veja na Figura 6, como ficaram as medidas da escala construída.

Figura 6 - Medidas da escala em centímetros do gráfico de barras construído pelo Sujeito 5 da turma A do Módulo III

\begin{tabular}{|c|c|}
\hline Frequência percentual & Centímetros \\
\hline 72 & 7,2 \\
\hline 11 & 1,1 \\
\hline 9 & 0,9 \\
\hline 7 & 0,7 \\
\hline 1 & 0,1 \\
\hline
\end{tabular}

$\mathrm{O}$ rigor apresentado na adequação da escala nos surpreendeu, sobretudo por ter sido feito por um estudante do Módulo III, grupo com desempenho menor que os estudantes com maior escolaridade. Entretanto, esse desempenho representa somente $1 \%$ dos dados analisados. Alguns estudantes consideraram a natureza proporcional dos valores das frequências e a partir disso realizaram uma representação grosseira da proporcionalidade da escala; outros estudantes, a maioria deles, apenas ordenou a disposição das barras por seriação decrescente. Assim sendo, decidimos analisar estas duas produções separadamente, pois nos revelaram graus de dificuldades quanto à inabilidade de construir uma escala, parecendo ser mais fácil apenas ordenar as barras do que tentar representar a natureza proporcional entre os valores das frequências. Primeiramente, analisaremos os gráficos que demonstraram tentativas de representar a proporcionalidade da escala, ou seja, os que diferenciaram as alturas das barras, mesmo não havendo qualquer atenção à precisão entre os intervalos.

Verificamos que $16 \%$ dos gráficos de barras construídos apresentaram a disposição da altura das barras respeitando as 
diferenças entre os valores das suas frequências. Percebemos ainda que esta estratégia variou em função dos dados apresentados em cada tarefa, sendo mais fácil representar a natureza proporcional entre as frequências na atividade de construção C-1 do que C-2. Observe na Figura 7, o gráfico de barras construído por um estudante do Módulo III em C-1.

Figura 7 - Gráfico de barras construído por um estudante do Módulo III. Sujeito 9 - Turma C - M III. Atividade C-1

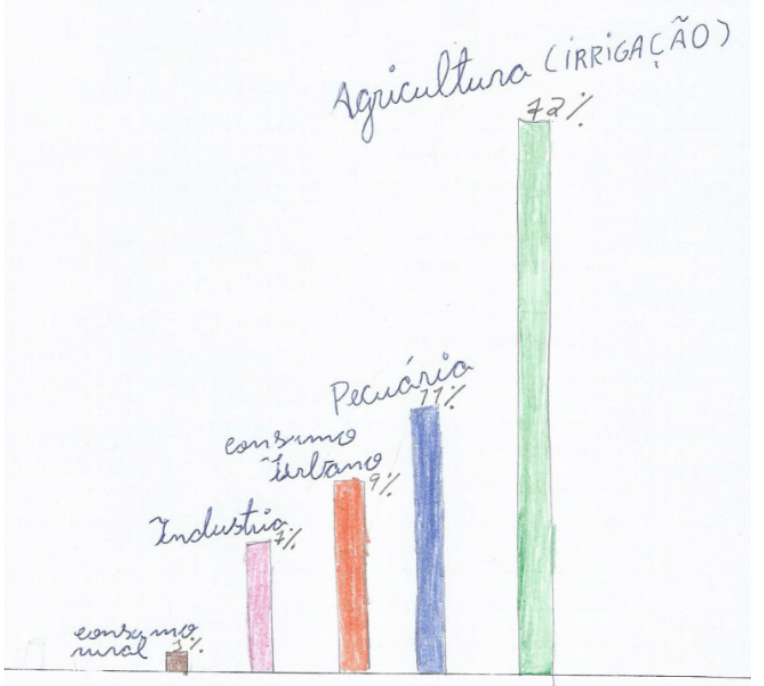

Observe que a estudante desenha a barra referente à categoria "Agricultura (irrigação)" bem mais alta que as três seguidas ("Pecuária", "Consumo urbano" e "Indústria"), estando ainda estas três dispostas mais próximas entre si (a diferença de frequências entre elas era menor do que em relação às duas outras barras), e por fim, a barra referente à categoria "Consumo rural" bem mais baixa que todas as outras. Esta foi uma estratégia apresentada em 23\% dos casos na atividade de construção $\mathrm{C}$-1, enquanto que na atividade de construção C-2 a tentativa de representar a natureza proporcional entre as frequências foi observada em 10\% dos casos. Observe a Figura 8.

Figura 8 - Gráfico de barras construído por um estudante do Módulo III. Sujeito 9 - Turma C - M III. Atividade C-2

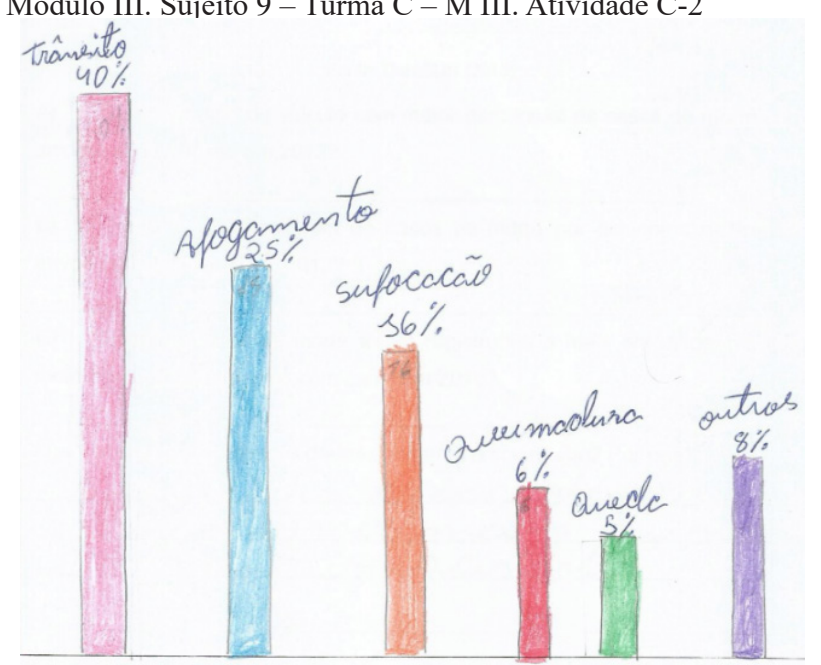

Observe que a estudante delimita, ainda que grosseiramente e sem recorrer à régua, mesmo tendo sido estimulada a utilizá-la, as alturas das barras comparando os valores das frequências. Observe ainda que as três últimas barras estão mais próximas entre si, tentando respeitar a pequena diferença entre os seus valores percentuais.

Uma justificativa provável para as diferenças na estratégia de representação da natureza proporcional entre C-1 e C-2 pode ter relação com os valores das frequências. Na atividade C-1 o valor da primeira frequência era bem maior que as demais e as seguintes eram próximas entre si, favorecendo alguns estudantes a se preocuparem em diferenciar esta barra das demais (ainda que não realizassem qualquer estimativa de proporcionalidade), enquanto que a atividade C-2 que apresentava os valores mais próximos uns dos outros, dificultando a representação destas diferenças. Outro fator que pode ter contribuído para esta estratégia em C1 é o fato da tabela utilizada para construir o gráfico já apresentar os dados em ordem decrescente, ainda que isso não fosse suficiente para que houvesse uma consideração maior entre a relação proporcional entre as frequências.

É pertinente destacar que, embora avaliar a natureza proporcional das frequências tenha sido uma estratégia considerável para definir as alturas das barras, mas aquém da habilidade de calibrar adequadamente uma escala, o percentual de gráficos apresentando esta estratégia foi baixo. Por outro lado, $57 \%$ dos gráficos construídos apresentaram as alturas das barras dispostas apenas por ordenação, seriadas na decrescente, assim como indicadas na tabela da tarefa.

A estratégia de ordenação por seriação decrescente estava presente independente da atividade de construção proposta. Entretanto, as análises seguintes serão feitas por atividade de construção, pois observamos dificuldades específicas em torno desta estratégia na atividade de C-2 comparada à atividade de $\mathrm{C}-1$.

Observamos que na atividade C-1 (Distribuição do uso da água no Brasil) todos os valores percentuais estavam em ordem decrescente, assim, os estudantes seguiam a sequência desenhando as barras em tamanho decrescente, estratégia observada em $49 \%$ dos gráficos construídos, como pode ser visto no exemplo da Figura 9.

Figura 9 - Gráfico de barras construído por um estudante do Módulo V. Sujeito 16 - Turma D - M V. Atividade C-1

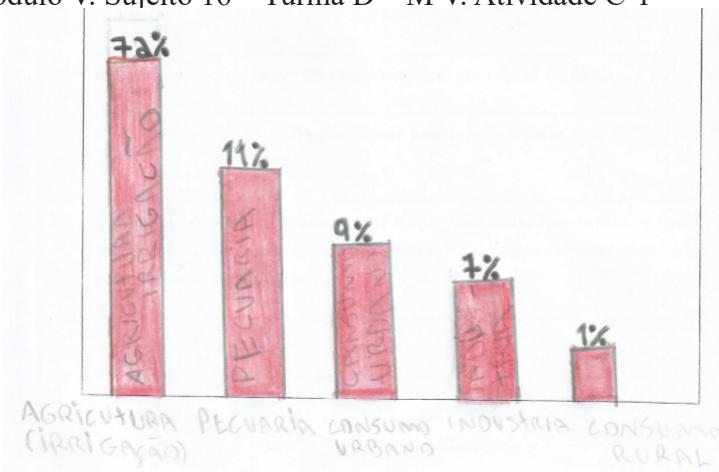


Podemos sugerir que seguir a tabela apresentada, na qual todos os valores já estavam em ordem decrescente, pode ter facilitado a ordenação da disposição das barras. Observe, ainda, que não há uma adequação do intervalo entre essas barras, estando dispostas como em degraus de escada.

A atividade de construção de gráfico de barras a partir da tabela "Mortalidade por acidentes" (C-2), entretanto, apresentava os valores percentuais quase todos em ordem decrescente, exceto o último que era maior que os dois valores que o antecediam, assim, os estudantes teriam que decidir entre organizar em seriação decrescente indicando o último dado apresentado na tabela na quarta posição no gráfico de barras ou copiar a sequência de apresentação dos dados da tabela, mas ajustando a seriação das barras em função do valor no momento de construir o gráfico. Os exemplos a seguir ajudam a compreender estas diferenças.

Figura 10 - Gráfico de barras construído por um estudante do Módulo III. Sujeito 3 - Turma C - M III. Atividade C-2

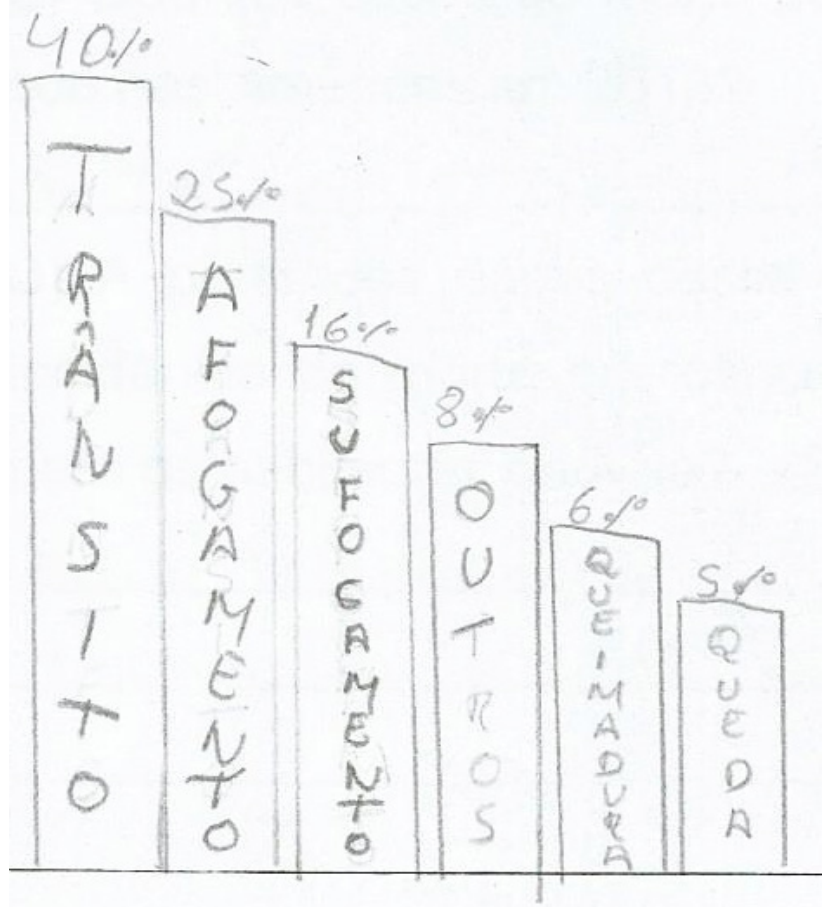

Observe que o estudante organiza as barras em ordem decrescente, mas ajusta a seriação das frequências no momento de desenhar as barras, colocando a categoria "8\%-Outros" entre as categorias "16\%-Sufocação" e "6\%-Queimaduras". Porém, sem adequação do intervalo entre essas barras, que estão apenas arrumadas por ordem. Também observamos estudantes que copiavam a seriação dos dados apresentados na tabela, mas ajustavam a altura da barra quando percebiam que embora a categoria " $8 \%$-Outros" estivesse por última era obrigatoriamente maior que a categoria " $6 \%$-Queimaduras" e “5\%-Queda”. O exemplo na Figura 11 é um desses casos.
Figura 11- Gráfico de barras construído por um estudante do Módulo III. Sujeito 10 - Turma C - M III. Atividade C-2

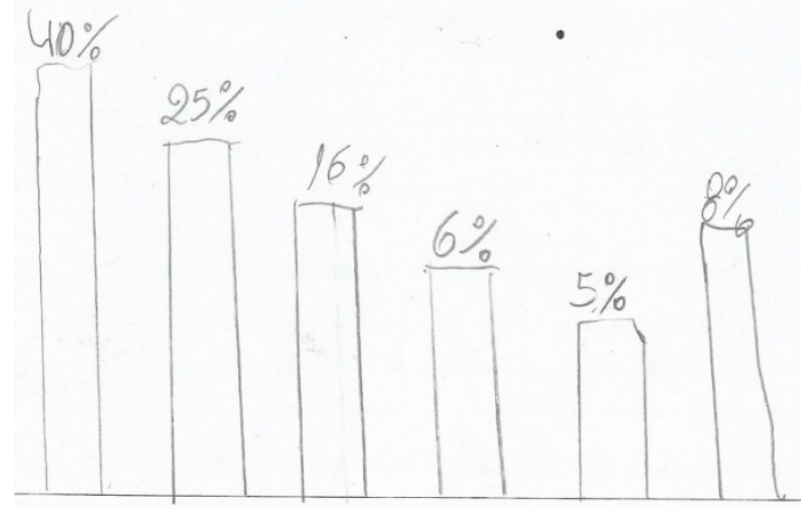

Podemos observar que a seriação dos dados apresentados na tabela foi mantida, mas houve a necessidade de reorganizar a altura da última barra pelo fato dela ter maior valor em comparação as duas anteriores (5\% e 6\%). Entretanto, quando os estudantes seriavam todas as barras em ordem decrescente, seguindo o modelo apresentado na tabela, sem se dar conta que a última frequência era maior que as duas antecedentes, alguns acabavam cometendo erros na representação dos dados, com o intuito de manter o tamanho decrescente das barras, como pode ser visto na Figura 12.

Figura 12 - Gráfico de barras construído por um estudante do Módulo III. Sujeito 5 - Turma A - M III. Atividade C-2

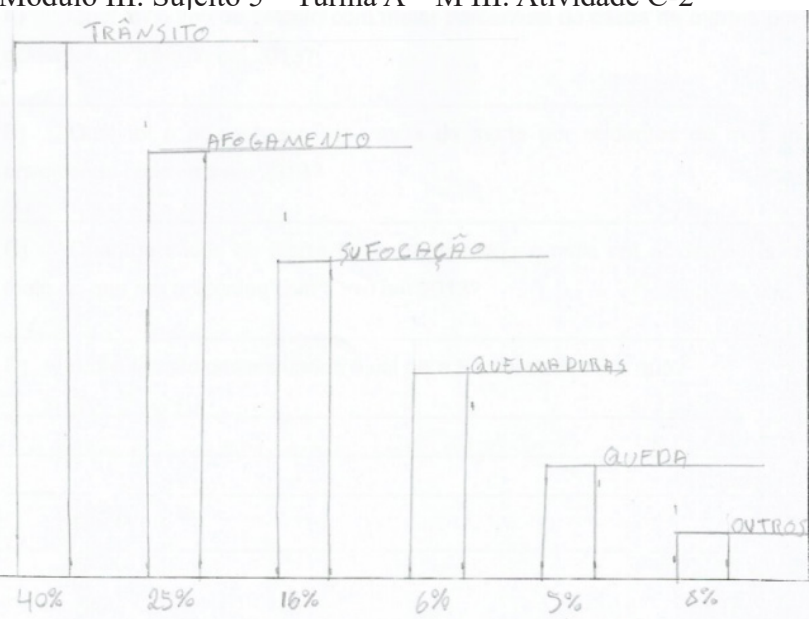

Observe que a categoria " $8 \%$-Outros" está mais baixa que as categorias " $6 \%$-Queimaduras" e " $5 \%$-Queda". Ou seja, para manter a seriação decrescente, o estudante cometeu uma inadequação na representação da frequência da última barra. Este tipo de inadequação esteve presente em 53\% dos casos.

Os resultados parecem indicar a necessidade um trabalho mais consistente e sistematizado em sala de aula voltado para questões fundamentais da construção de uma representação gráfica refletindo-se, sobretudo, a inclusão de elementos básicos necessários para a transmissão da informação e a adequação da escala. Dificuldade com a escala adotada foi um dos aspectos mais evidentes entre os estudantes e neste sentido podemos afirmar que o papel do professor é extremamente 
importante para auxiliar os estudantes a refletirem sobre a construção proporcional dos valores da escala.

\section{Conclusão}

Apesar das dificuldades identificadas pelos estudantes da EJA na construção correta de gráficos de barras, verificamos efeito positivo da escolaridade. Ou seja, testes de análise de variância (ANOVA) indicaram efeito significativo da escolaridade nos resultados gerais em construção tendo os estudantes do Módulo $\mathrm{V}$ construído mais gráficos quando solicitados do que o grupo de estudantes com menor escolaridade. A escolaridade também favoreceu o aprimoramento dos gráficos de barras construídos, na medida em que elementos estruturantes foram incluídos mais vezes, houve mais gráficos iniciados por uma linha de base e alguma estratégia de diferenciação entre a altura das barras foi utilizada mais vezes pelos estudantes do Módulo $\mathrm{V}$ do que pelos estudantes do Módulo III.

Diferentemente do que foi observado em Lima (2010), em que não se observou efeito positivo da escolarização por estudantes da EJA ao longo da Educação Básica, os dados encontrados neste estudo sinalizaram diferenças importantes entre os segmentos da EJA no Ensino Fundamental, convergindo com a argumentação levantada pelo Instituto Paulo Montenegro e Ação Educativa (2016, p. 8) de que conforme maior escolaridade, maior é o desempenho na escala de alfabetismo e de que as dificuldades entre os que concluem a primeira etapa do Ensino Fundamental é bastante elevado (nossos resultados também revelaram desempenhos inferiores por parte dos estudantes com menor tempo de escolarização).

Embora a escolaridade tenha se apresentado como fator positivo, não podemos deixar de chamar atenção ao fato de que muitos jovens e adultos ainda não conseguem construir gráficos de barras corretamente, o que representa um grande desafio para a escola, pois de acordo com os documentos curriculares oficiais é esperado que este público seja capaz de coletar, organizar, apresentar e interpretar dados, ler, compreender e construir gráficos de barras, de linhas e de setores e tabelas desde o final da primeira etapa do Ensino Fundamental. (Brasil, 2001; Brasil, 2002; Pernambuco, 2012). Porém, os resultados deste estudo revelaram que, mesmo completando a segunda etapa do Ensino Fundamental, muitas dificuldades foram identificadas na construção de gráficos de barras.

Entretanto, é importante que os jovens e adultos desenvolvam habilidades para representar informações estatísticas e para comunicar suas reações diante de tais informações, tornando-se capazes de compreender um determinado assunto, baseado em conjuntos de dados estatisticamente tratados; pensar criticamente em torno de tais resultados; expressar opiniões; fazer inferências; tomar decisões e familiarizar-se com informações estatísticas oficiais como aspecto relevante para o desenvolvimento do letramento estatístico e para a vida em sociedade.

\section{Referências}

Albuquerque, M.R.G.C. (2010). Como adultos e crianças compreendem a escala representada em gráficos. Recife: Universidade Federal de Pernambuco.

Alcântara, L.R. (2012). O ensino de conteúdos estatísticos no Projovem Campo - Saberes da terra em Pernambuco. Recife: Universidade Federal de Pernambuco.

Brasil. Ministério da Educação e Desporto. (2001). Educação para jovens e adultos: ensino fundamental: proposta curricular para o primeiro segmento. São Paulo: Ação Educativa. Brasília, DF, Brasil. Brasília: MEC.

Brasil. Ministério da Educação e Desporto. (2002). Educação para jovens e adultos: ensino fundamental: proposta curricular para o segundo segmento: $5^{a} a 8^{a}$ série. São Paulo: Ação Educativa. Brasília, DF, Brasil. Brasília: Ministério da Educação e Desporto.

Brasil. Ministério da Educação. (2017). BNCC - Base Nacional Comum Curricular: educação é a base. Brasília: MEC.

Cazorla, I.M., \& Santana, E. (2010). Do Tratamento da Informação ao Letramento Estatístico. Itabuna: Via Litterarum.

Friel, S.N., Curcio, F.R., \& Bright, G.W. (2001). Making Sense of Graphs: Critical Factors Influencing Comprehension and Instructional Implications. Journal for Research in Mathematics Education, 32(2). 124-158.

Gal, I. (2002). Adults 'Statistical Literacy: Meanings, Components, Responsibilities. International Statistical Review, 7 0. 1. 1-5 1.

Garfield, J. (2002). The Challenge of Developing Statistical Reasoning. Journal of Statistics Education, 10(3). 1-12.

Instituto Paulo Montenegro e Ação Educativa. (2016). Indicador de alfabetismo funcional - INAF: estudo especial sobre alfabetismo e mundo do trabalho. São Paulo, SP, Brasil.

Lima, I. B. (2010). Investigando o desempenho de jovens $e$ adultos na construção e interpretação de gráficos (Dissertação de Mestrado). Centro de Educação, Universidade Federal de Pernambuco, Recife, PE, Brasil.

Martins, M. N. P., \& Carvalho, C. F. (2018). O ensino de gráficos estatísticos nos anos iniciais. Rencima: Revista de Ensino de Ciências e Matemática, 9(2). 247-264.

Morais, P. C. C. C. (2010). Construção, leitura e interpretação de gráficos estatísticos por alunos do 9. ${ }^{\circ}$ ano de escolaridade (Dissertação de Mestrado). Universidade do Minho, Portugal.

Pernambuco. Secretaria de Educação. (2012). Parâmetros para a Educação Básica do Estado de Pernambuco: Parâmetros Curriculares de Matemática Educação de Jovens e Adultos. Recife, PE, Brasil.

Santos, C. C. (2014). Possibilidades do uso do computador no ensino de gráficos: um estudo em escolas do ProJovem Recife (Dissertação de Mestrado). Centro de Educação, Universidade Federal de Pernambuco, Recife, PE, Brasil.

Soek, A. M., Haracemiv, S. M. C., \& Stoltz, T. (2009). Mediação pedagógica na alfabetização de jovens e adultos. Curitiba: Ed. Positivo. 